\title{
Analysis of Power Factor and Voltage Regulation in Large Industrial Enterprises
}

\author{
Wei $\mathrm{Li}^{1}$, Changcheng Zhou ${ }^{2}$, Jianhua Yang ${ }^{2}$, a , Lei Huang ${ }^{3}$, Fan Zhang ${ }^{1}$ \\ ${ }^{1}$ Beijing Electric Power Economic Research Institute, Beijing 100055, China \\ ${ }^{2}$ College of Information and Electrical Engineering, China Agricultural University, Beijing 100083 , \\ China \\ ${ }^{3}$ State Grid Corporation Beijing Electric Company, Beijing 100031, China \\ ayang.haag@163.com
}

Keywords: Power factor, reactive power compensation, voltage regulation, transformer tap changer, enterprise power network

\begin{abstract}
Improvement of power factor can reduce resistive power losses, increase the bus voltage and in many cases improve motor performance in an industrial enterprise power network. The regulating elements implemented in the network include transformer tap changers, generators regulators, static shunt capacitors. The capability of the elements is investigated and analyzed in compensating the power factor and maintaining the voltage in a suitable range. The process to find out the range at bus bars is introduced for voltage regulation. Some efficient cases are presented and selected for power factor and voltage regulation in the network, which is effective to large industrial enterprises.
\end{abstract}

\section{Introduction}

As energy travels from generators to customers, electrical resistance in power transformers and lines causes dissipation of energy. Lower power factor results in inefficient power use and wasted energy, which should be corrected whenever possible. The reactive power generated by generators is limited in power systems, the needed reactive power in a distribution network or an industrial enterprise power network should be supplied by local compensation [1]. Capacitor banks can provide local complementary reactive power that decrease these reactive power movements and, consequently, reduce power losses [2]. An additional benefit from decreasing the amount of reactive power that flows in the networks is improvement in voltage profiles [3]. Power factor regulation and voltage regulation should be considered together since there is a mutual dependence between the reactive power flow and the voltage profile.

Based on the calculation and analysis of the power network data in an industrial enterprise, the power factor regulation and the voltage regulation are considered in this paper. Some efficient methods are applied for investigating capability of the regulating elements, such as transformer tap changers, generators regulators and capacitor banks, in compensating the power factor and maintaining the voltage in a suitable range. The process to find out this range at bus bars is displayed and introduced for voltage regulation. Load flow studies are done in order to determine the voltage variation at the bus bars with the prospective operating cases.

\section{Reactive Power of Electrical Equipment}

The loss of reactive power in conductors and transformers can be computed by applying some reduced formulas [2]. The effect of inductive reactance and capacitive admittance of cables can be determined as the follow equation. In small load currents, the cable produces reactive power due to its capacitive reactance, but as the load current increases, the cable may be a consumer of reactive power. 


$$
\left.\begin{array}{l}
\Delta Q_{\text {ind }}=3(2 \pi f L) I \\
\Delta Q_{\text {cap }}=2 \pi f C U_{n}^{2} \\
\Delta Q=\Delta Q_{\text {ind }}-\Delta Q_{\text {cap }}
\end{array}\right\}
$$

Where $\Delta Q$ is reactive power loss of cables, $U_{\mathrm{n}}$ nominal system voltage, $f$ nominal frequency, $C$ capacitance of cables, $L$ inductance of cables. $C$ and $L$ can be gotten from some cable manuals [4].

The reactive power loss of a transformer can be calculated according to the below equation.

$$
\Delta Q_{T}=\frac{I_{0} \% S_{n T}}{100}+\frac{U_{k} \% S_{n T}}{100}\left(\frac{S_{\text {Load }}}{S_{n T}}\right)^{2}
$$

Where $\Delta Q_{\mathrm{T}}$ is reactive power loss of a transformer, $U_{\mathrm{k}} \%$ percentage of short-circuit voltage drop of the transformer, $S_{\mathrm{nT}}$ total apparent power of the transformer, $S_{\text {load }}$ total apparent power of load, $I_{0} \%$ percentage of open-circuit current of the transformer.

\section{Network Configuration and Voltage Regulation}

The single line diagram of the enterprise power network is shown in figure 1 . The $35 \mathrm{kV}$ network could be fed via one or two 110/36.6 kV transformer(s) from the power network, POWNET, which belongs to a local power company. The considered maximum load demand is $50 \mathrm{MW}$ in the enterprise network. Two turbo generators, $2 \times 25 \mathrm{MW}$, with voltage or power factor regulation can be connected to $35 \mathrm{kV}$ bus bar, NS. Medium-voltage motors are on the $6 \mathrm{kV}$ main bus bars as individual motors. Lumped low-voltage motors on the secondary side of the $6 / 0.4 \mathrm{kV}$ distribution transformers. There are capacitors banks both at $35 \mathrm{kV}$ and $6 \mathrm{kV}$ bus bars.

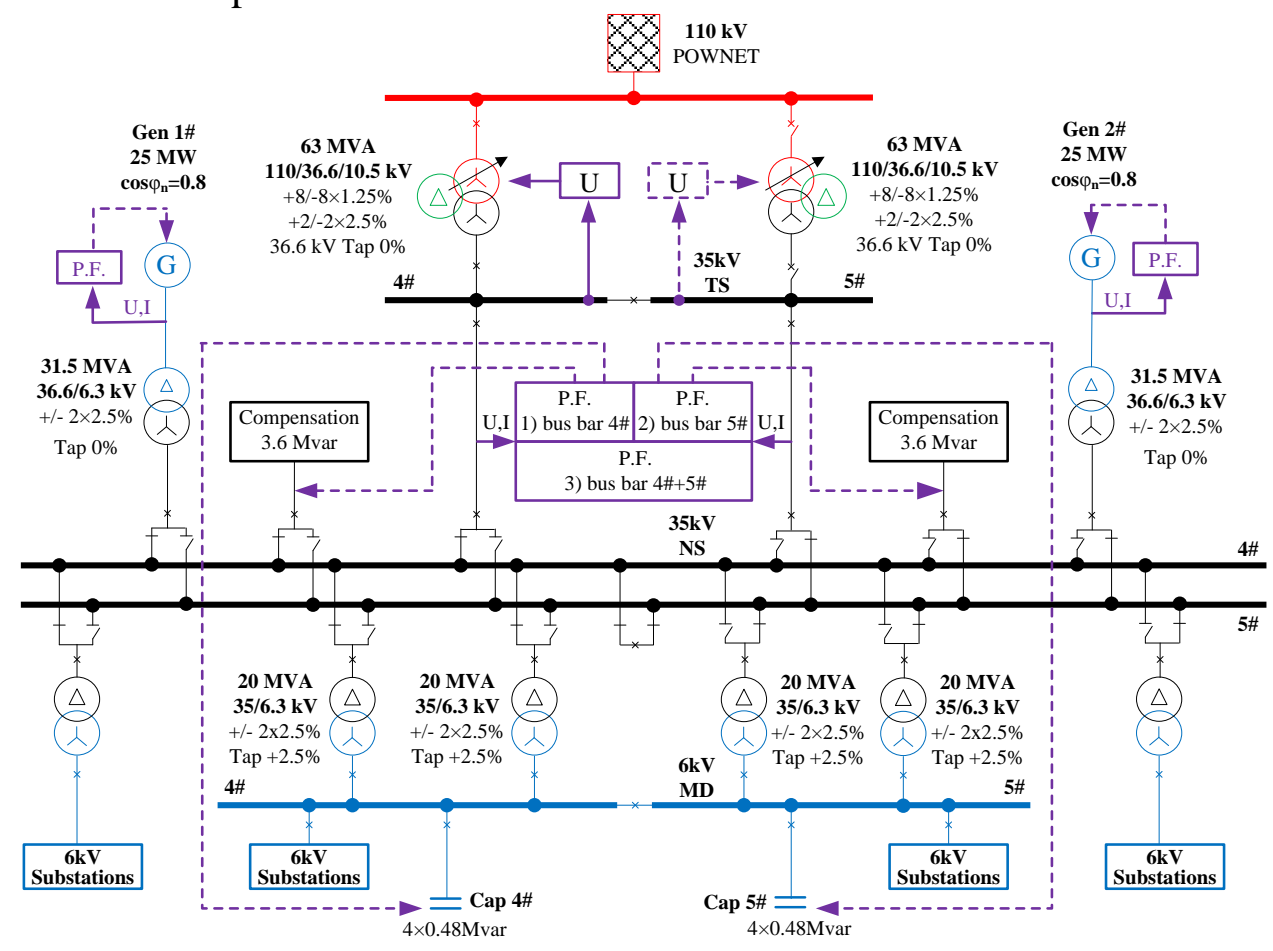

Fig.1 Single line diagram of enterprise network

The voltage regulation and the power factor regulation should be considered together since there is a mutual dependence between voltage and reactive power flow. Therefore, a control strategy is necessary so that the control elements can work not against each other, and the network elements cannot be overloaded at a steady state.

Combination of different methods can be implemented for voltage control purpose. The most popular equipments for the voltage regulation are generators, tap-changing transformers and capacitor banks. There are some boundary conditions which have to be met for the regulation. For example, the voltage at NS must be regulated in a certain range in order to achieve a constant voltage at $6 \mathrm{kV}$ bus bars. Since all $6 \mathrm{kV}$ substations are supplied via 35/6.3 kV transformers with 
fixed tap positions, the voltages at $6 \mathrm{kV}$ can only be adjusted together by increasing or decreasing the voltage at NS, which can be adjusted with the tap changers of the 110/36.6/10.5 kV transformers that have on-load tap changers at $110 \mathrm{kV}$ with 17 tap positions and a range of $+8 /-8 \times 1.25 \%$.

The operating range of the voltage at NS is limited by some operating conditions. In the case of maximum load at 35/6.3 $\mathrm{kV}$ distribution transformer, i.e. operation with a single transformer and closed bus coupling at $6 \mathrm{kV}$, the voltage drop caused by the transformer impedance reaches the maximum value. The minimum voltage at NS can allow operation with about $93 \% U_{\mathrm{n}}$ at $6 \mathrm{kV}$ bus bars. In the case of no load at $6 \mathrm{kV}$ substations, i.e. starting of production after shut down, the maximum voltage at NS can allow operation with about $107 \% U_{\mathrm{n}}$ at $6 \mathrm{kV}$ bus bars [5]. In the case of maximum active and reactive power output of a generator, the minimum voltage at NS can allow operation of the generator with about $105 \% U_{\mathrm{n}}$. In the case of no power output of a generator, the maximum voltage at NS can allow operation of the generator with about $95 \% U_{\mathrm{n}}$.

The possible voltage range at NS is calculated and its result is shown in Table 1 . Therefore, the voltage at NS should be in the range of $98.9 \%$ to $102.6 \% U_{\mathrm{n}}$ in order to fulfill all the conditions at 6 $\mathrm{kV}$. Similar, the voltage at $110 \mathrm{kV}$ bus bar, TS, should be in a range of $96.8 \%$ to $109.2 \% U_{\mathrm{n}}$ to fulfil a voltage deviation from -1.1 to $+2.6 \% U_{\mathrm{n}}$ at $35 \mathrm{kV}$ with the on-load tap changers of the 110/36.6/10.5 kV transformers.

Table 1 Voltage Range at NS (35 kV) to Keep Voltage Deviations of +/-7 \% at 6 kV Substations and +/-5 \% at 6.3 kV Generators

\begin{tabular}{|c|c|c|c|c|c|c|c|c|c|c|}
\hline \multirow{2}{*}{ Transformer } & \multirow{2}{*}{$\begin{array}{c}\text { Tap Pos. } \\
\quad / \%\end{array}$} & $I_{\text {Load }} / I_{\mathrm{nT}} / \%$ & \multirow[t]{2}{*}{0} & \multicolumn{5}{|c|}{100} & \multirow{2}{*}{ Max. } & \multirow{2}{*}{ Min. } \\
\hline & & Power Factor & & 1.00 & 0.95 & 0.90 & 0.85 & 0.80 & & \\
\hline \multirow{2}{*}{ Dist. Transf. 35/6.3 kV } & \multirow{2}{*}{2.5} & $U_{\min 35 \mathrm{kV}} / \%$ & 92.9 & 93.9 & 96.7 & 97.7 & 98.5 & 98.9 & \multirow[t]{2}{*}{98.9} & \\
\hline & & $U_{\max 35 \mathrm{kV}} / \%$ & 102.8 & 103.7 & 106.1 & 106.9 & 107.6 & 108.0 & & 102.8 \\
\hline \multirow{2}{*}{ Gen. Transf. 36.6/6.3 kV } & \multirow{2}{*}{0} & $U_{\min 35 \mathrm{kV}} / \%$ & 97.7 & 97.5 & 94.3 & 93.1 & 92.2 & 91.5 & \multirow[t]{2}{*}{97.7} & \\
\hline & & $U_{\max 35 \mathrm{kV}} / \%$ & 108.0 & 107.6 & 105.0 & 103.8 & 103.0 & 102.6 & & 102.6 \\
\hline
\end{tabular}

The power flow via a transformer causes a voltage drop which increases with higher utilization $\left(S_{\text {load }} / S_{\mathrm{nT}}\right.$ or $\left.I_{\text {load }} / I_{\mathrm{nT}}\right)$ of the transformer, higher transformer impedance and lower power factor. The voltage drop, $\Delta U \%$, depends mainly on $U_{\mathrm{k}} \%$ of the transformer and the reactive power flow via the transformer according to the below equation $[2,6]$.

$$
\Delta U \%=\frac{S_{\text {Load }}}{S_{n T}}\left(U_{r} \% \cos \varphi_{\text {load }}+U_{x} \% \sin \varphi_{\text {load }}\right) \approx \frac{Q_{\text {Load }}}{S_{n T}} U_{k} \%
$$

The voltage at TS can also be adjusted by changing the reactive power flow via the $110 / 36.6 / 10.5 \mathrm{kV}$ transformer. On the other side, increasing and decreasing of the reactive power flow via the transformer always changes the power factor at the connection point to POWNET, which is not desired.

Although the generators are equipped with a voltage regulator, the voltage regulation cannot be done only with the generators because of their operating limits at leading power factor. If the voltage regulation is activated, any voltage fluctuation at NS may cause a change of the reactive power output of the generators. The voltage fluctuations at NS can be caused by the voltage fluctuations in POWNET, by changing of the tap position of 110/36.6/10.5 kV transformers or by switching on and off of the loads, e.g. start-up of a motor. If the generators work in parallel operation with voltage regulation, the voltage fluctuations at NS would also change the reactive power output of the generators and the power factor at connection point to POWNET. In addition, the voltage regulators of the generators and the tap changer of the 110/36.6/10.5 kV transformer can work against each other. Since the voltage regulation of any generator is faster than one of the tap changer, the generator should normally try at first to compensate voltage fluctuations which can lead to overloading of a generator at a steady state. Therefore, if the voltage at NS is regulated by the voltage controller of the tap changer of the 110/36.6/10.5 kV transformers, the generators should work with constant power factor or reactive power output. If both 110/36.6/10.5 kV transformers are in operation and TS or NS bus bars are coupled, the voltage controllers of the transformers have to be coordinated, i.e. master/follower operation mode. 


\section{Power Factor and Reactive Power Regulation}

A generator can generally work with constant power factor or reactive power output if its excitation system has an additional control feature besides voltage regulation. The power factor or reactive power controller is often implemented as an overlaid controller of the voltage controller which changes the voltage reference value slowly. The advantage of such controlling structure is that in the cases of voltage drop, e.g. short-circuit or start-up of a motor, the generator immediately supply the necessary reactive power in the first seconds and the reactive power regulator can only adjust the operating point for the steady state condition, i.e. after 1 minute.

Capacitor banks can be applied at the individual motors and distribution panels. In the enterprise network, the capacitors banks for power factor regulation are connected to both $35 \mathrm{kV}$ and $6 \mathrm{kV}$ voltage levels, which can be used to control the power factor (P.F.) to TS for parallel operation and as fixed compensation to support the generators in isolated operation.

The regulation tasks of tap-changing transformers, generators and capacitor banks are summarized in Table 2 for parallel and isolated operation.

Table 2 Task of voltage and power factor regulator equipment

\begin{tabular}{ccc}
\hline Voltage and P. F. Control Equipment & Parallel Operation & Isolated Operation \\
\hline $110 / 36.6 / 10.5 \mathrm{kV}$ tap-changing transformer & $\mathrm{U}$ & $\mathrm{U}$ \\
Gen 1\# & P.F. & U or P.F. \\
Gen 2\# & P.F. & const. Q \\
Capacitors at NS $(35 \mathrm{kV})$ & P.F. to TS & const. Q \\
Cap 4\# & P.F. to TS & const. Q \\
Cap 5\# & P.F. to TS &
\end{tabular}

In the case of operation with open bus coupling at NS to busbar 4\# and 5\#, two independent power factor controllers for the capacitors are necessary. If the bus bars at NS are coupled and both incoming feeders of NS are in operation, the power factor controllers for the capacitors have to be coordinated to avoid circulating reactive current, as shown in figure 1, and a third power factor controller will be used.

In isolated operation and closed bus bar coupling at NS, one generator must be responsible for voltage regulation. For open bus bar coupling at NS one generator for each bus bar is necessary. In the case of decoupling the generator should be changed from power factor regulation to voltage regulation. If it is necessary, the capacitor banks can be used as fixed compensation to support the generator with reactive power supply.

\section{Conclusion}

The power network data in an industrial enterprise is displayed and analyzed. The process to find out the possible voltage range at bus bars is introduced for voltage regulation. Load flow studies are done in order to determine the voltage variation at 35 and $6 \mathrm{kV}$ bus bars with the prospective operating cases. The tasks of regulating equipments are determined in the voltage control and the power factor control, which include generators regulators, transformer tap changers and capacitor banks. The analysis method is effective and may be applied to enterprise power networks and distribution networks.

\section{Acknowledgements}

This work is financially supported by the Science and Technology Project of SGCC (PD17201200045).

\section{References}

[1] S. Bisanovic, M. Hajro, M. Samardzic. One approach for reactive power control of capacitor banks in distribution and industrial networks [J]. Electrical Power and Energy Systems, 2014, (60): 67-73.

[2] Jianhua Yang. Steady-state Analysis and Economic Operation of Power Systems [M]. Beijing: China Electric Power Press, 2013 (In Chinease). 
[3] S. X. Chen, Y. S. Foo. Eddy, H. B. Gooi, et al. A Centralized Reactive Power Compensation System for LV Distribution Networks [J]. IEEE Trans. on Power Systems, 2015, 30(1): 274-284.

[4] L. Heinhold, R. Stubbe. Power Cables and Their Applications (3rd Edition) [M]. Weinheim: Wiley-VCH Verlag GmbH, 1993.

[5] GB/T 12325-2008 Power quality - Deviation of supply voltage [S].

[6] A. L. Sheldrake. Handbook of Electrical Engineering [M]. West Sussex: John Wiley \& Sons Ltd, 2003 